\title{
Synthesis, Characterization, and Evaluation of Biological Activities of Imidazolyl-Isoxazolime Analogue
}

\author{
Narayana U. Kudva ${ }^{1}{ }^{\mathbb{D}}$, Sumana Y. Kotian ${ }^{1, * \mathbb{D}}$, Vrushabendra Basavanna ${ }^{2} \mathbb{D}$, Vicas C.S $^{3(\mathbb{D})}$, \\ Satish K. Byrappa ${ }^{1}(\mathbb{D})$, Shridevi $D^{4(\mathbb{D})}$, Srikantamurthy Ningaiah ${ }^{2}(\mathbb{D})$, Turuvekere Krishnamurthy Chaitra \\ 5 (iD), Srinivasa Murthy V 6(D), K. Byrappa ${ }^{3}$ iD , K. M. Lokanatha Rai 1,*(D) \\ 1 Department of Studies in Chemistry, University of Mysore, Manasagangotri, Mysuru-570 005, Karnataka, India, Email: \\ nuke.org.chem@gmail.com; sumanakotian@gmail.com; bsathishorg@gmail.com; kmlrai@yahoo.com; \\ 2 Department of Chemistry, Vidyavardhaka College of Engineering, Mysore-570 002, Karnataka, India, Email: \\ srijmn@gmail.com; vrushabendra@vvce.ac.in; \\ 3 Department of Studies in Earth Science, University of Mysore, Manasagangotri, Mysuru-570 005, Karnataka, India, Email: \\ csvicas@gmail.com, kbyrappa@gmail.com; \\ 4 Chemical Sciences and Technology Division, CSIR-NIIST, Thiruvananthapuram-695 019, Kerala, India, Email: \\ shridevi20@gmail.com; \\ 5 Department of Studies in Chemistry, Pooja Bhagavat Memorial Mahajana PG Centre, KRS Road, Mysuru-570 016, \\ Karnataka, India, Email: chaitratk.chemistry@ gmail.com; \\ 6 Department of Chemistry, Dayanand Sagar University, Bangalore- 560078, Karnataka, India, Email: \\ vsrinivasamurthy3429@gmail.com; \\ * Correspondence: sumanakotian@gmail.com (S.Y.K); kmlrai@yahoo.com (K.M.L.R);
}

Received: 2.06.2020; Revised: 30.05.2020; Accepted: 2.06.2020; Published: 7.06.2020

\begin{abstract}
Synthesis of isoxazoline heterocycle containing benzimidazole moiety with the highest bioactivity using substituted benzaldehyde as starting material is reported in this paper. In the beginning, the precursor benzaldehydes were treated with hydroxylamine hydrochloride to afford respective aldoximes. The resultant compound was subjected to cyclization reaction with allyl chloride in the presence of chloramine-T to afford isoxazoline key intermediate. Finally, benzimidazole was subjected to $S$-alkylation with isoxazoline moiety to afford the title compound with good yield. This method cultivated many advantages like; short reaction time and easy isolation. All the compounds structurally characterized by ${ }^{1} \mathrm{H}$ NMR, ${ }^{13} \mathrm{C}$ NMR, LCMS, IR spectral data, and elemental analysis. Besides, all the synthesized compounds were tested for their antimicrobial and antioxidant activity. The bioactivity was envisioned that the compound $\mathbf{5 a}$ and $\mathbf{5} \mathbf{f}$ exhibited excellent antifungal activity, which may be helpful in developing a lead to inhibit microbes.
\end{abstract}

Keywords: Isoxazoline; benzimidazole; chloramine-T; allyl chloride; antimicrobial.

(C) 2020 by the authors. This article is an open-access article distributed under the terms and conditions of the Creative Commons Attribution (CC BY) license (https://creativecommons.org/licenses/by/4.0/).

\section{Introduction}

Isoxazolines [1] are five-membered unsaturated heterocycles containing three carbon atoms, one nitrogen, and oxygen atoms (adjacent) to each other. Heterocycles containing nitrogen and oxygen atom are considered as useful scaffolds for the synthesis of biologically active molecules and liquid crystals [2-4]. Among such classes of compounds, isoxazolines occupy a prime position in bioorganic chemistry for their diverse biological applications. They have been reported to exhibit anticancer [5], antimalarial [6], antimicrobial [7], antioxidant [8], anthelminthic [9], enzyme inhibition [10] and insecticidal activities [11]. Isoxazolines also serves as an essential building block in organic synthesis [12]. 
The benzo derivative of imidazole: benzimidazole and its derivatives have received considerable attention after it was found to be a part of Vitamin $\mathrm{B}_{12}$ [13]. Benzimidazoles are truly versatile [14], as compounds that have a benzimidazole core are known to have a variety of biological activities such as antiviral [15], anticancer [16], analgesic [17], anthelminthic [18], anti-inflammatory [19], antitumor [20], antibacterial and antifungal activity [21]. Most of the biologically active benzimidazole derivatives are known to be modified at positions 1-,2and 5- (or 6-) by various substituents. Modifying the benzimidazole nucleus has thus resulted in anthelmintics [22] (albendazole, mebendazole, thiabendazole) and also many lead compounds in a wide range of therapeutic areas. In particular, mercaptobenzimidazole is used in the synthesis of omeprazole, lansoprazole, and pantoprazole [23], which are protein pump inhibitors and are used as antiulcerous agents in the treatment of stomach and duodenal ulcers [24]. Thus, we have synthesized a series of compounds incorporating two potent heterocyclesisoxazoline and benzimidazole in a single scaffold and to evaluate their biological activity.

\section{Materials and Methods}

The chemical ingredients, viz., para bromo benzaldehyde, para nitro benzaldehyde, veratraldehyde, anisaldehyde, benzaldehyde, piperonal, allyl chloride were purchased from LOBA Chemie, India. Hydroxylamine hydrochloride was obtained from SRL, India. Sodium acetate, Potassium Carbonate, and Diethyl ether were procured from RANKEM, India. Ethanol was purchased from CHANGSHU YANGYUAN CHEMICAL, China. The proposed structure ofthe intermediate compounds and that of the final compound were confirmed by the ${ }^{1} \mathrm{H}$ and ${ }^{13} \mathrm{C}-\mathrm{NMR}$ spectra obtained using an AGILENT (400 MHz) NMR spectrometer (Deuterated chloroform as solvent procured from SIGMA ALDRICH, USA and tetramethylsilane as internal standard). The following representations denoted the peak types in the spectra: singlet (s), doublet (d), doublet of doublets (dd), triplet (t), quartet (q), and multiplet (m). Infrared spectra (IR) were obtained using a Perkin Elmer spectrophotometer. The ${ }^{1} \mathrm{H}$ and ${ }^{13} \mathrm{C}$-NMR and LCMS spectra were used for the confirmation of the molecular structure, hydrogen bonding, and the purity of the sample. Antibacterial property and antifungal property of the given compounds was assessed using pure cultures of Escherichia coli (MTCC - 443), Pseudomonas aeruginosa (MTCC - 2453), Staphylococcus aureus (MTCC - 3610), Bacillus subtilis (MTCC - 10619), Enterococcus faecalis (MTCC - 439), Aspergillus niger (MTCC - 3323) and Candida albicans (MTCC - 227), procured from Microbial Type Culture Collection (MTCC) and Gene Bank, Chandigarh, India. Penicillin [25] and Streptomycin were used as standards. The experiment was performed in well plates, and plates were scanned using a microplate reader (Thermo scientific, Multiskan ${ }^{\mathrm{TM}}$ ) at 580-600 $\mathrm{nm}$ after the incubation period. Absorbance reading was taken at $517 \mathrm{~nm}$ and $541 \mathrm{~nm}$ using UV-Vis spectrophotometer $\left(\right.$ ELICO $^{\circledR}$ SA 165 , India) for the assessment of antioxidant activity using DPPH assay and Haemolysis assay, respectively.

\section{Results and Discussion}

The structures of newly synthesized compounds $\mathbf{3 a}$ and $\mathbf{5 a}$ were confirmed by their analytical and other spectral data. In the ${ }^{1} \mathrm{H}$ NMR spectra of compound 3a, three protons $\left(-\mathrm{CH}_{2}\right.$ and $-\mathrm{CH}$ ) of the isoxazoline ring were seen as a doublet of the doublet. Among three protons, $-\mathrm{CH}$ is the most deshielded one due to its proximity to oxygen and oxadiazole moiety and it 
appeared as a doublet of doublets. The methylene protons of the isoxazoline ring $\left(-\mathrm{CH}_{2}\right)$, exhibited a typical ABX spin system with $-\mathrm{CH}$ as a doublet of doublets.

The ${ }^{1} \mathrm{H}$ NMR spectra of the compound 5a showed peaks at $\delta 3.10-3.20(\mathrm{dd}, 1 \mathrm{H}), 3.63-$ $3.66(\mathrm{dd}, 1 \mathrm{H}), 4.59-4.63(\mathrm{dd}, 1 \mathrm{H})$ similarly, the appearance of singlet due to $-\mathrm{CH}_{2}-$ group of isoxazoline 5a at $\delta 5.40$ and also disappearance of peaks at $\delta 12.10(1 \mathrm{H}, \mathrm{s})$ due to $-\mathrm{SH}$ proton confirms the formation of product $\mathbf{5 a}$.

In ${ }^{13} \mathrm{C}$ NMR spectra, the appearance of peaks at $\delta 37.8-\mathrm{CH}_{2}$ of and $65.8-\mathrm{CH}$ of isoxazoline and also the appearance of a peak at 34.7 of compound $\mathbf{5 a}$ instead of a peak at 45.1 for $\mathrm{CH}_{2} \mathrm{Cl}$ of the compound 3a confirms the formation of the title compound. The mass spectrum of all the compounds showed a molecular ion peak at $[\mathrm{M}+1]$ corresponding to its molecular formula, which confirmed its chemical structure.

\subsection{Experimental.}

3.1.1. Scheme of synthesis.

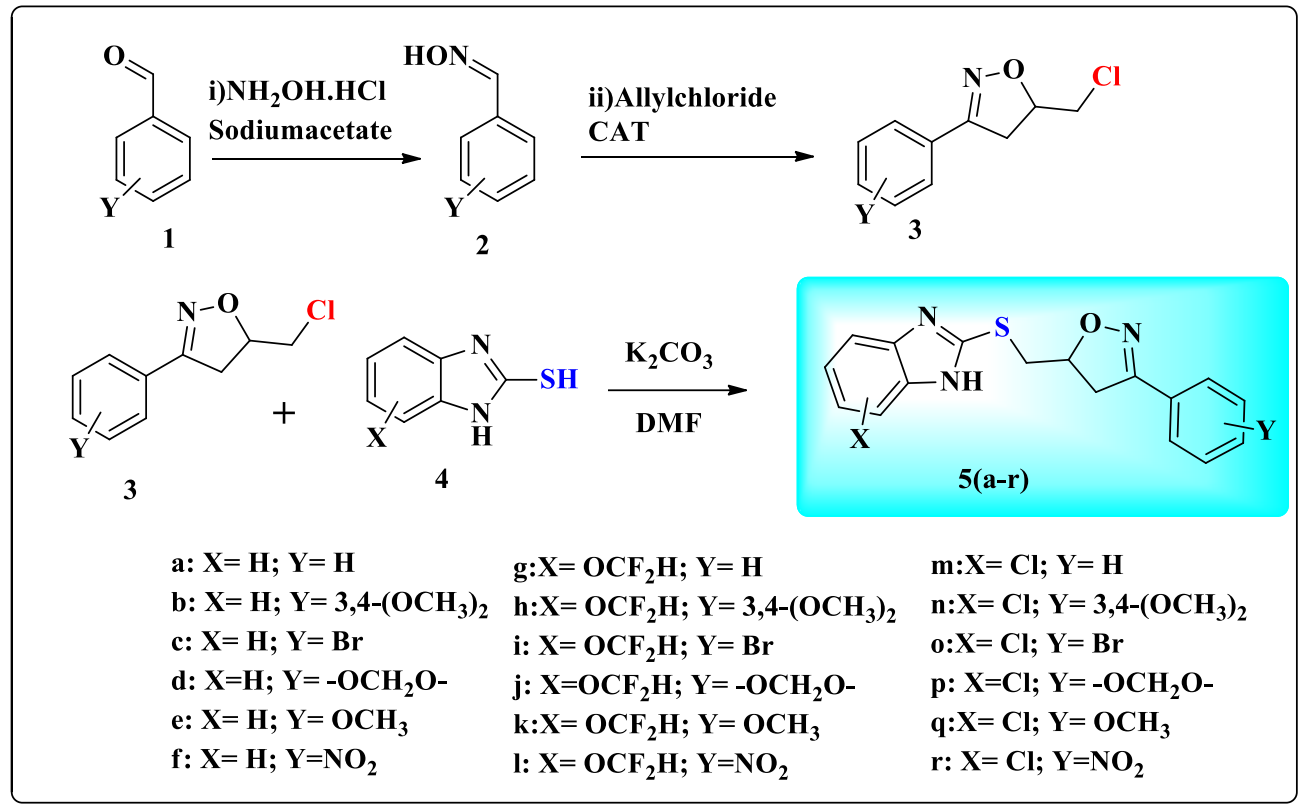

Scheme 1. Synthesis of Imidazolyl-isoxazoline derivative.

\subsubsection{General procedure for the synthesis of aromatic aldoximes.}

About $1-1.5 \mathrm{~g}$ benzaldehyde $(1 \mathrm{mmol})$ in $15 \mathrm{ml}$ ethanol was added to a solution of hydroxylamine hydrochloride $(1.5 \mathrm{mmol})$ and sodium acetate $(1.5 \mathrm{mmol})$ the mixture was heated at $80-90^{\circ} \mathrm{C}$ for $1 \mathrm{~h}$ and then it was allowed to cool to room temperature. The precipitate was collected and was taken to the next step.

\subsubsection{General procedure for the synthesis of the isoxazoline derivative.}

Allyl chloride (1mmol), respective aldoxime (1mmol), and chloramine-T (1.2 mmol) in ethanol $(20 \mathrm{~mL})$ were taken in a reaction flask, and it was stirred for 8 hours at room temperature $25^{\circ} \mathrm{C}$. The progress of the reaction was monitored by TLC. After completion, Sodium chloride formed was filtered off and washed with ethanol $(15 \mathrm{~mL})$. Filtrate and washing were combined, and the solvent was evaporated in a vacuum. The residue was extracted with ether $(25 \mathrm{~mL} \times 3)$. The extract was washed successively with water $(15 \mathrm{~mL} \times 2)$, 
$10 \% \mathrm{NaOH}(15 \mathrm{~mL} \times 2)$, and saturated brine solution $(10 \mathrm{~mL})$. The organic layer was dried over anhydrous $\mathrm{Na}_{2} \mathrm{SO}_{4}$. The crude product was filtered and purified by recrystallization using methanol. The probable mechanism is shown in scheme 2.

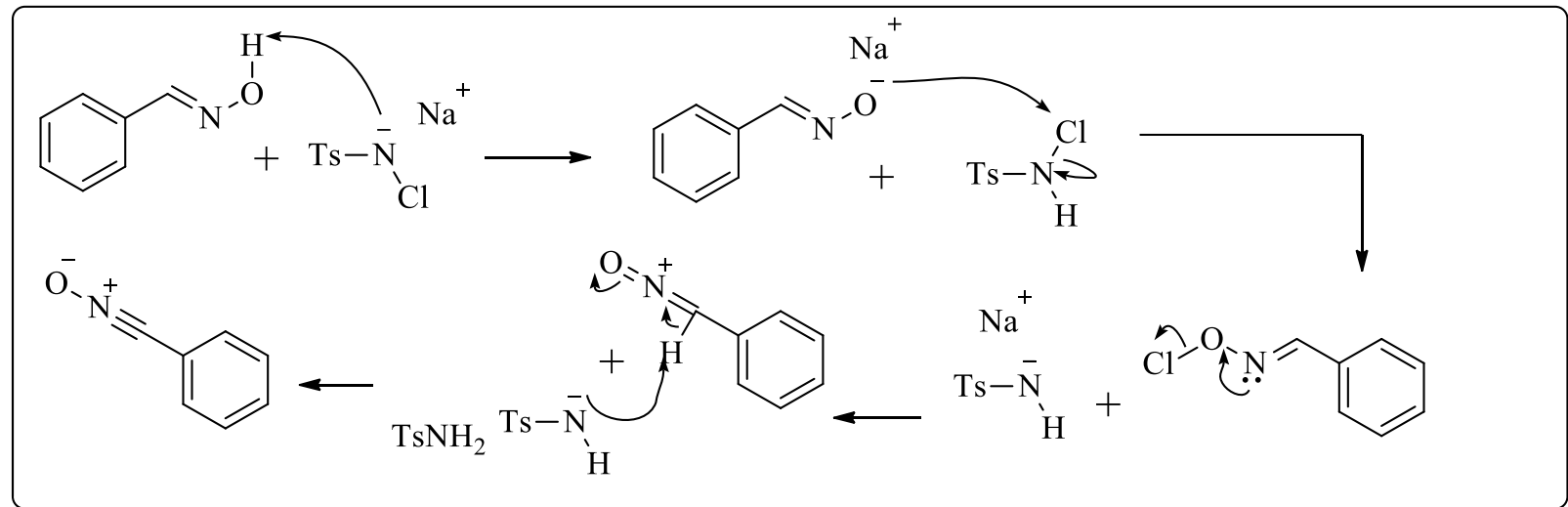

Scheme 2. The possible mechanism for the generation of nitrile oxides using chloramine-T.

3.1.4. General procedure for $\mathrm{N}$-alkylation.

Respective isoxazoline derivative $(1 \mathrm{mmol})$ and respective benzimidazole derivative (1 $\mathrm{mmol})$ and $\mathrm{K}_{2} \mathrm{CO}_{3}(3 \mathrm{mmol})$ and dimethylformamide were stirred for 8 hours at room temperature. The solid product was extracted into the ether layer, and it was dried over anhydrous $\mathrm{Na}_{2} \mathrm{SO}_{4}$ (Scheme 1).

5-[\{(1H-benzo[d]imidazol-2-yl)thio\}methyl]-3-phenyl-4,5-dihydroisoxazole(5a): Prepared from 3a and 4a. White solid, Yield: 78\%. ${ }^{1} \mathrm{H}$ NMR (400MHz, DMSO): $\delta$ 12.55(s, 1H), 7.65(m, 2H, ArH), 7.46(m, 2H, ArH), 7.10(d, 1H, ArH), 7.09(m, 2H, ArH), 5.10(m, 1H), 3.59(m, 3H), 3.50(m, 2H). ${ }^{13} \mathrm{C}$ NMR (100MHz, DMSO): $\delta 156.2,147.1,138.9,136.0,131.0$, 128.8, 128.2, 123, 115.6, 70.4, 45.0, 39.9. Anal. Calcd for $\mathrm{C}_{17} \mathrm{H}_{15} \mathrm{~N}_{3} \mathrm{OS}: \mathrm{C}-66.00 ; \mathrm{H}-4.89 ; \mathrm{N}$ - 13.58\%; Found: C - 65.87; H - 4.76; N - 13.42\%. LCMS [M+1]: Calcd for $\mathrm{C}_{17} \mathrm{H}_{15} \mathrm{~N}_{3} \mathrm{OS}$ : 310.09, Found: 310.11.

5-[\{(1H-benzo[d]imidazol-2-yl)thio\}methyl]-3-(3,4-dimethoxyphenyl)-4,5dihydroisoxazole (5b): Prepared from $3 \mathbf{b}$ and $\mathbf{4 b}$. White solid, Yield: 76\%. ${ }^{1} \mathrm{H}$ NMR $(400 \mathrm{MHz}$, DMSO): $\delta 12.55$ (s, 1H), 7.59 (m, 1H, ArH), 7.44 (d, 2H, ArH), $7.40(\mathrm{~m}, 1 \mathrm{H}, \operatorname{ArH}), 7.13$ (d, 2H, ArH), 6.98 (m, 1H, ArH), $3.9(\mathrm{~m}, 2 \mathrm{H}), 3.85(\mathrm{~s}, 3 \mathrm{H}), 3.83(\mathrm{~s}, 3 \mathrm{H}), 3.19$ - $2.94(\mathrm{~m}, 2 \mathrm{H}), 2.98$ - 2.73 (m, 2H). ${ }^{13} \mathrm{C}$ NMR (100MHz, DMSO): $\delta$ 156.2, 152.1, 149.9, 147.1, 138.9, 127.3, 123.0, 121.3, 114.3, 115.2, 111.9, 70.4, 56.2, 45.0, 39.9. Anal. Calcd for $\mathrm{C}_{19} \mathrm{H}_{19} \mathrm{~N}_{3} \mathrm{O}_{3} \mathrm{~S}: \mathrm{C}-61.77 ; \mathrm{H}-$ 5.18; N - 11.37\%; Found: C - 61.64; H - 5.06; N - 11.16\%. LCMS [M+1]: Calcd for $\mathrm{C}_{19} \mathrm{H}_{19} \mathrm{~N}_{3} \mathrm{O}_{3} \mathrm{~S}: 370.44$, Found: 370.56 .

5-[\{(1H-benzo[d]imidazol-2-yl)thio\}methyl]-3-(4-bromophenyl)-4,5-dihydroisoxazole $(5 c)$ : Prepared from 3c and 4c. White solid, Yield: 75\%. ${ }^{1} \mathrm{H}$ NMR (400MHz, DMSO): $\delta 12.55$ (s, 1H), 7.70 (d, 2H, ArH), 7.64 (d, 2H, ArH), 7.44 (d, 2H, ArH), 7.13 (d, 2H, ArH), 3.9 (m, $2 \mathrm{H}), 3.19-2.98$ (m, 2H), $2.94-2.73$ (m, 2H). ${ }^{13} \mathrm{C}$ NMR (100MHz, DMSO): $\delta 156.2,147.2$, 138.9, 131.7, 129.4, 128.6, 125.4, 123.0, 115.2, 70.4, 45.0, 39.9. Anal. Calcd for $\mathrm{C}_{17} \mathrm{H}_{14} \mathrm{BrN}_{3} \mathrm{OS}$ : $\mathrm{C}-52.59 ; \mathrm{H}-3.63 ; \mathrm{N}-10.82 \%$; Found: $\mathrm{C}-52.15 ; \mathrm{H}-3.14 ; \mathrm{N}-10.38 \%$. LCMS [M+1]: Calcd for $\mathrm{C}_{17} \mathrm{H}_{14} \mathrm{BrN}_{3} \mathrm{OS}$ : 389.28 , Found: 389.36.

5-[\{(1H-benzo[d]imidazol-2-yl)thio\}methyl]-3-(benzo[d][1,3]dioxol-5-yl)-4,5-

dihydroisoxazole (5d): Prepared from 3d and 4d. White solid, Yield: 72\%. ${ }^{1} \mathrm{H}$ NMR $(400 \mathrm{MHz}$, DMSO): $\delta 12.55$ (s, 1H), 7.59 (m, 1H, ArH), 7.44 (d, 2H, ArH), $7.40(\mathrm{~m}, 1 \mathrm{H}, \operatorname{ArH}), 7.13$ (d, 2H, ArH), 6.98 (m, 1H, ArH), 6.06 (s, 2H), $3.9(\mathrm{~m}, 2 \mathrm{H}), 3.19$ - $2.94(\mathrm{~m}, 2 \mathrm{H}), 2.98-2.73$ (m, 
2H). ${ }^{13} \mathrm{C}$ NMR (100MHz, DMSO): $\delta$ 156.2, 151.1, 148.9, 147.2, 138.9, 127.3, 123.0, 121.0, 115.3, 114.2, 111.9, 101.2, 70.4, 45.0, 39.9. Anal. Calcd for $\mathrm{C}_{18} \mathrm{H}_{15} \mathrm{~N}_{3} \mathrm{O}_{3} \mathrm{~S}: \mathrm{C}-61.18 ; \mathrm{H}-4.28$; $\mathrm{N}-11.89 \%$; Found: $\mathrm{C}-61.42 ; \mathrm{H}-4.21 ; \mathrm{N}-11.46 \%$. LCMS [M+1]: Calcd for $\mathrm{C}_{18} \mathrm{H}_{15} \mathrm{~N}_{3} \mathrm{O}_{3} \mathrm{~S}$ : 354.39, Found: 354.41.

5-[\{(1H-benzo[d]imidazol-2-yl)thio\}methyl]-3-(4-methoxyphenyl)-4,5dihydroisoxazole (5e):Prepared from 3e and 4e. White solid, Yield: 76\%. ${ }^{1} \mathrm{H}$ NMR $(400 \mathrm{MHz}$, DMSO): $\delta$ 1H NMR: 12.57 (s, 1H),7.57 (d, 2H, ArH), 7.48 (d, 1H, ArH), 7.32 (d, 1H, ArH), $7.01(\mathrm{~d}, 2 \mathrm{H}, \mathrm{ArH}), 6.97(\mathrm{~d}, 2 \mathrm{H}, \mathrm{ArH}), 5.02(\mathrm{~s}, 1 \mathrm{H}), 3.58(\mathrm{~s}, 3 \mathrm{H}), 3.54(\mathrm{~m}, 3 \mathrm{H}), 3.50(\mathrm{~m}, 1 \mathrm{H}) .{ }^{13} \mathrm{C}$ NMR (100MHz, DMSO): $\delta$ 162.9, 156.2, 147.1, 138.9, 128.7, 123, 122, 115.4, 114.2, 70.4, 55.8, 45, 39.9. Anal. Calcd for $\mathrm{C}_{18} \mathrm{H}_{17} \mathrm{~N}_{3} \mathrm{O}_{2} \mathrm{~S}: \mathrm{C}-63.70 ; \mathrm{H}-5.05 ; \mathrm{N}-12.38 \%$; Found: C - 63.62; $\mathrm{H}-5.01 ; \mathrm{N}-12.14 \%$. LCMS [M+1]: Calcd for $\mathrm{C}_{18} \mathrm{H}_{17} \mathrm{~N}_{3} \mathrm{O}_{2} \mathrm{~S}: 340.10$, Found: 340.24.

5-[\{(1H-benzo[d]imidazol-2-yl)thio\}methyl]-3-(4-nitrophenyl)-4,5-dihydroisoxazole (5f):Prepared from 3f and 4f. Yellow solid, Yield: 72\%. ${ }^{1} \mathrm{H}$ NMR (400MHz, DMSO): $\delta 12.54$ (s, 1H), 8.34 (d, 2H, ArH), 8.03 (d, 2H, ArH), 7.44 (d, 2H, ArH), 7.13 (d, 2H, ArH), 3.9 (m, 2H), 3.19 - 2.98 (m, 2H), $2.94-2.78$ (m, 2H). ${ }^{13} \mathrm{C}$ NMR (100MHz, DMSO): $\delta 156.2,150.2$, 147.1, 138.9, 136.5, 127.7, 123.0, 115.2, 70.4, 45.0, 39.9. Anal. Calcd for $\mathrm{C}_{17} \mathrm{H}_{14} \mathrm{~N}_{4} \mathrm{O}_{3} \mathrm{~S}: \mathrm{C}-$ 57.62; H - 3.98; N - 15.81\%; Found: C - 57.43; H - 3.09; N - 15.22\%. LCMS [M+1]: Calcd for $\mathrm{C}_{17} \mathrm{H}_{14} \mathrm{~N}_{4} \mathrm{O}_{3} \mathrm{~S}: 355.38$, Found: 355.56 .

5-[\{(6-(difluoromethoxy)-1H-benzo[d]imidazol-2-yl)thio\}methyl]-3-phenyl-4,5dihydroisoxazole (5g): Prepared from 3g and 4g. White solid, Yield: 70\%. ${ }^{1} \mathrm{H}$ NMR (400MHz, DMSO): $\delta 12.73$ (s, 1H), 7.57 (s, 1H, ArH), 7.32 (s, 1H), 7.14 (d, 1H, ArH), 6.96 (s, 4H), 5.02 $(\mathrm{m}, 1 \mathrm{H}), 3.77$ (s, 3H), 3.53 (d, 2H,), 3.23 (s, 1H). ${ }^{13} \mathrm{C}$ NMR (100MHz, DMSO): $\delta$ 167.3, 156.2, 156.1,147.2, 139.9, 136.0, 131.2, 130.0, 128.2, 116.2, 111.5, 100.8, 70.4, 45.0, 39.9. Anal. Calcd for $\mathrm{C}_{18} \mathrm{H}_{15} \mathrm{~F}_{2} \mathrm{~N}_{3} \mathrm{O}_{2} \mathrm{~S}: \mathrm{C}-57.59 ; \mathrm{H}-4.03 ; \mathrm{N}-11.19 \%$; Found: $\mathrm{C}-57.51 ; \mathrm{H}-4.01 ; \mathrm{N}$ 11.02\%. LCMS [M+1]: Calcd for $\mathrm{C}_{18} \mathrm{H}_{15} \mathrm{~F}_{2} \mathrm{~N}_{3} \mathrm{O}_{2} \mathrm{~S}: 376.39$, Found: 376.45 .

5-[\{(6-(difluoromethoxy)-1H-benzo[d]imidazol-2-yl)thio\}methyl]-3-(3,4-

dimethoxyphenyl)-4,5-dihydroisoxazole (5h): Prepared from $\mathbf{3 h}$ and $\mathbf{4 h}$. White solid, Yield: 71\%. ${ }^{1} \mathrm{H}$ NMR (400MHz, DMSO): $\delta 12.57$ (s, 1H), $7.49(\mathrm{~m}, 2 \mathrm{H}), 7.40(\mathrm{~s}, 1 \mathrm{H}), 7.35(\mathrm{~m}, 1 \mathrm{H})$, $7.15(\mathrm{~s}, 1 \mathrm{H}), 6.92(\mathrm{~m}, 2 \mathrm{H}), 5.02(\mathrm{~m}, 1 \mathrm{H}), 3.80(\mathrm{~s}, 6 \mathrm{H}), 3.21(\mathrm{dd}, 1 \mathrm{H}), 3.11(\mathrm{~m}, 2 \mathrm{H}), 2.97(\mathrm{dd}$, 1H). ${ }^{13} \mathrm{C}$ NMR (100MHz, DMSO): $\delta$ 167.3, 156.1, 156.2, 152.1, 149.9, 147.1, 139.9, 131.2, 127.3, 121.0, 116.2, 114.3, 111.9, 111.5, 100.8, 70.4, 56.3, 45.0, 39.9. Anal. Calcd for $\mathrm{C}_{20} \mathrm{H}_{19} \mathrm{~F}_{2} \mathrm{~N}_{3} \mathrm{O} 4 \mathrm{~S}$ : C - 55.17; H - 4.40; N - 9.65\%; Found: C - 55.16; H - 4.02; N - 9.44\%. LCMS $[\mathrm{M}+1]$ : Calcd for $\mathrm{C}_{20} \mathrm{H}_{19} \mathrm{~F}_{2} \mathrm{~N}_{3} \mathrm{O}_{4} \mathrm{~S}: 436.44$, Found: 463.57 .

3-(4-bromophenyl)-5-[\{(6-(difluoromethoxy)-1H-benzo[d]imidazol-2-yl)thio\}methyl]4,5-dihydroisoxazole (5i): Prepared from 3i and 4i. White solid, Yield: 74\%. ${ }^{1} \mathrm{H}$ NMR (400MHz, DMSO): $\delta 12.55$ (s, 1H), 7.70 (d, 2H, ArH), 7.64 (d, 2H, ArH), 7.56 (m, 1H, ArH), 7.36 (s, 2H), 7.12 (m, 1H, ArH), 6.93 (m, 1H,ArH), 3.90 (m, 2H) 3.19 - 2.98 (m, 2H), 2.94 $2.73(\mathrm{~m}, 2 \mathrm{H}) .{ }^{13} \mathrm{C}$ NMR (100MHz, DMSO): $\delta$ 167.3, 156.2, 156.1, 147.1, 139.9, 131.7, 131.2, 128.6, 127.6, 125.4, 116.2, 111.5, 100.8, 70.4, 45.0, 39.9. Anal. Calcd for $\mathrm{C}_{18} \mathrm{H}_{14} \mathrm{BrF}_{2} \mathrm{~N}_{3} \mathrm{O}_{2} \mathrm{~S}$ : C - 47.59; H - 3.11; N - 9.25\%; Found: C - 47.32; H - 3.24; N - 9.36\%. LCMS [M+1]: Calcd for $\mathrm{C}_{18} \mathrm{H}_{14} \mathrm{BrF}_{2} \mathrm{~N}_{3} \mathrm{O}_{2} \mathrm{~S}$ : 455.29, Found: 455.34 .

3-(benzo[d][1,3]dioxol-5-yl)-5-[\{(6-(difluoromethoxy)-1H-benzo[d]imidazol-2-

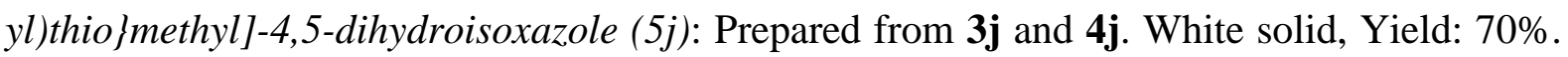
${ }^{1} \mathrm{H}$ NMR (400MHz, DMSO): $\delta 12.56$ (s, 1H), 7.59 (m, 1H, ArH), 7.56 (m, 1H, ArH), 7.40 (m, $1 \mathrm{H}, \mathrm{ArH}), 7.35(\mathrm{~m}, 1 \mathrm{H}, \mathrm{ArH}), 6.98(\mathrm{~m}, 1 \mathrm{H}, \mathrm{ArH}), 6.93(\mathrm{~m}, 1 \mathrm{H}, \mathrm{ArH}), 6.06(\mathrm{~s}, 2 \mathrm{H}), 3.91(\mathrm{~m}$, 2H), $3.19-2.94$ (m, 2H), $2.98-2.73$ (m, 2H). ${ }^{13} \mathrm{C}$ NMR (100MHz, DMSO): $\delta$ 167.3, 156.2, 
156.1, 151.1, 148.9, 147.2, 139.9, 131.2, 127.3, 121.0, 116.3, 114.3, 111.9, 101.3, 100.8, 70.4,45, 39.9. Anal. Calcd for $\mathrm{C}_{19} \mathrm{H}_{15} \mathrm{~F}_{2} \mathrm{~N}_{3} \mathrm{O}_{4} \mathrm{~S}$ : C - 54.41; H - 3.60; N - 10.02\%; Found: C 54.24; $\mathrm{H}-3.42 ; \mathrm{N}-10.42 \%$. LCMS [M+1]: Calcd for $\mathrm{C}_{19} \mathrm{H}_{15} \mathrm{~F}_{2} \mathrm{~N}_{3} \mathrm{O}_{4} \mathrm{~S}$ : 420.40, Found: 420.57.

5-[\{(6-(difluoromethoxy)-1H-benzo[d]imidazol-2-yl)thio\}methyl]-3-(4-

methoxyphenyl)-4,5-dihydroisoxazole (5k): Prepared from 3k and 4k. White solid, Yield: 75\%. ${ }^{1} \mathrm{H}$ NMR (400MHz, DMSO): $\delta 12.57$ (s, 1H), 7.90 (d, 2H), $7.49(\mathrm{~m}, 3 \mathrm{H}), 7.40(\mathrm{~s}, 1 \mathrm{H}), 7.35$ (m, $1 \mathrm{H}), 7.10(\mathrm{~d}, 1 \mathrm{H}), 5.02(\mathrm{~m}, 1 \mathrm{H}), 3.83(\mathrm{~s}, 3 \mathrm{H}), 3.20(\mathrm{dd}, 1 \mathrm{H}), 3.10(\mathrm{~m}, 2 \mathrm{H}), 2.95(\mathrm{dd}, 1 \mathrm{H}),{ }^{13} \mathrm{C}$ NMR (100MHz, DMSO): $\delta 167.3,162.9,156.2,156.1,147.1,139.9,131.2,128.7,122,116.2$, 114.4,111.5, 100.8, 70.4, 55.7, 45, 39.9. Anal. Calcd for $\mathrm{C}_{19} \mathrm{H}_{17} \mathrm{~F}_{2} \mathrm{~N}_{3} \mathrm{O}_{3} \mathrm{~S}$ : C - 56.29; H - 4.23; $\mathrm{N}-10.36 \%$; Found: C - 56.65; H - 4.23; N - 10.65\%. LCMS [M+1]: Calcd for $\mathrm{C}_{19} \mathrm{H}_{17} \mathrm{~F}_{2} \mathrm{~N}_{3} \mathrm{O}_{3} \mathrm{~S}$ : 406.42, Found: 406.48.

5-[\{(6-(difluoromethoxy)-1H-benzo[d]imidazol-2-yl)thio\}methyl]-3-(4-nitrophenyl)4,5-dihydroisoxazole (5l): Prepared from 31 and 4l. Yellow solid, Yield: $72 \% .{ }^{1} \mathrm{H}$ NMR (400MHz, DMSO) $\delta: 12.56(\mathrm{~s}, 1 \mathrm{H}), 8.34$ (d, 2H, ArH), 8.07 (d, 2H, ArH), 7.56 (m, 1H, ArH), $7.36(\mathrm{~s}, 2 \mathrm{H}), 7.12(\mathrm{~m}, 1 \mathrm{H}, \mathrm{ArH}), 6.93(\mathrm{~m}, 1 \mathrm{H}, \mathrm{ArH}), 3.19$ - $2.94(\mathrm{~m}, 2 \mathrm{H}), 2.98-2.73(\mathrm{~m}, 2 \mathrm{H})$. ${ }^{13} \mathrm{C}$ NMR (100MHz, DMSO) $\delta: 167.3,156.1,156.2,150.2,147.3,139.9,136.5,131.2,127.7$, 116.2, 111.5, 100.8, 70.4, 45.0, 39.9. Anal. Calcd for $\mathrm{C}_{18} \mathrm{H}_{14} \mathrm{~F}_{2} \mathrm{~N}_{4} \mathrm{O}_{4} \mathrm{~S}: \mathrm{C}-51.43 ; \mathrm{H}-3.36 ; \mathrm{N}-$ 13.33\%; Found: C - 51.43; H - 3.11; N - 13.12\%. LCMS [M+1]: 421.39, Found: 420.41.

5-[\{(6-chloro-1H-benzo[d]imidazol-2-yl)thio\}methyl]-3-phenyl-4,5-dihydroisoxazole $(5 \mathrm{~m})$ : Prepared from $\mathbf{3 m}$ and $\mathbf{4 m}$. White solid, Yield: $71 \% .{ }^{1} \mathrm{H}$ NMR $(400 \mathrm{MHz}, \mathrm{DMSO})$ $\delta: 12.52(\mathrm{~s}, 1 \mathrm{H}), 8.40(\mathrm{~s}, 1 \mathrm{H}), 7.90(\mathrm{~d}, 2 \mathrm{H}), 7.53(\mathrm{~m}, 4 \mathrm{H}), 7.49(\mathrm{~d}, 1 \mathrm{H}), 5.05(\mathrm{~m}, 1 \mathrm{H}), 3.17(\mathrm{dd}, 1 \mathrm{H})$, $3.12(\mathrm{~m}, 2 \mathrm{H}), 2.90(\mathrm{dd}, 1 \mathrm{H}) .{ }^{13} \mathrm{C}$ NMR (100MHz, DMSO) $\delta: 156.2,147.1,140.3,137,136,131$, 129.2, 128.2, 124.1, 116.6, 115.8, 70.4, 45, 39.9. Anal. Calcd for $\mathrm{C}_{17} \mathrm{H}_{14} \mathrm{ClN}_{3} \mathrm{OS}: \mathrm{C}-59.38 ; \mathrm{H}$ - 4.10; N - 12.22\%; Found: C - 59.11; H - 4.01; N - 12.32\%. LCMS [M+1]: Calcd for $\mathrm{C}_{17} \mathrm{H}_{14} \mathrm{ClN}_{3} \mathrm{OS}$ : 344.06, Found: 344.01 .

5-[\{(6-chloro-1H-benzo[d]imidazol-2-yl)thio\}methyl]-3-(3,4-dimethoxyphenyl)-4,5dihydroisoxazole (5n): Prepared from 3n and 4n. White solid, Yield: 76\%. ${ }^{1} \mathrm{H}$ NMR $(400 \mathrm{MHz}$, DMSO): $\delta 12.56(\mathrm{~s}, 1 \mathrm{H}), 8.36(\mathrm{~s}, 1 \mathrm{H}, \operatorname{ArH}), 7.59(\mathrm{~m}, 1 \mathrm{H}, \operatorname{ArH}), 7.51(\mathrm{~m}, 1 \mathrm{H}, \operatorname{ArH}), 7.14(\mathrm{~m}$, 1H, ArH), 6.98 (m, 1H, ArH), 3.92 (m, 2H), 3.85 (s, 3H), 3.83 (s, 3H), 3.19 - $2.94(\mathrm{~m}, 2 \mathrm{H})$, 2.98 - $2.73(\mathrm{~m}, 2 \mathrm{H}) .{ }^{13} \mathrm{C}$ NMR (100MHz, DMSO): $\delta$ 167.3, 156.1, 156.2, 152.1, 149.9, 147.1, 139.9, 131.2, 127.3, 121,116.2, 114.3, 111.9, 111.5, 100.8, 70.4, 56.3, 45.0, 39.9. Anal. Calcd for $\mathrm{C}_{19} \mathrm{H}_{18} \mathrm{ClN}_{3} \mathrm{O}_{3} \mathrm{~S}: \mathrm{C}, 56.50 ; \mathrm{H}, 4.49 ; \mathrm{N}, 10.40 \%$; Found: C: $56.33, \mathrm{H}: 4.43 \mathrm{~N}: 10.61 \%$. LCMS [M+1]: Calcd for $\mathrm{C}_{17} \mathrm{H}_{14} \mathrm{ClN}_{3} \mathrm{OS}: 404.08$, Found: 404.15 .

3-(4-bromophenyl)-5-[\{(6-chloro-1H-benzo[d]imidazol-2-yl)thio\}methyl]-4,5dihydroisoxazole (5o): Prepared from 30 and 4o. White solid, Yield: 74\%. ${ }^{1} \mathrm{H}$ NMR $(400 \mathrm{MHz}$, DMSO): $\delta 12.56(\mathrm{~s}, 1 \mathrm{H}), 8.36(\mathrm{~m}, 1 \mathrm{H}, \mathrm{ArH}), 7.70(\mathrm{~d}, 2 \mathrm{H}, \mathrm{ArH}), 7.64(\mathrm{~d}, 2 \mathrm{H}, \mathrm{ArH}), 7.51(\mathrm{~m}$, $1 \mathrm{H}, \mathrm{ArH}), 7.14(\mathrm{~m}, 1 \mathrm{H}, \mathrm{ArH}), 3.90(\mathrm{~m}, 2 \mathrm{H}), 3.19-2.98(\mathrm{~m}, 2 \mathrm{H}), 2.94-2.73(\mathrm{~m}, 2 \mathrm{H}) .{ }^{13} \mathrm{C} \mathrm{NMR}$ (100MHz, DMSO): $\delta 156.2,147.1,140.3,137.0,131.7,129.4,129.2,128.6,124.1,115.8,70.5$, 45.0, 39.9. Anal. Calcd for $\mathrm{C}_{17} \mathrm{H}_{13} \mathrm{BrClN}_{3} \mathrm{OS}: \mathrm{C}-48.30 ; \mathrm{H}-3.10 ; \mathrm{N}-9.94 \%$; Found: $\mathrm{C}-48.21$; $\mathrm{H}-3.12 ; \mathrm{N}-9.45 \%$. LCMS [M+1]: Calcd for $\mathrm{C}_{17} \mathrm{H}_{13} \mathrm{BrClN}_{3} \mathrm{OS}: 424.96$, Found: 424.98.

3-(benzo[d][1,3]dioxol-5-yl)-5-[\{(6-chloro-1H-benzo[d]imidazol-2-yl)thio\}methyl]4,5-dihydroisoxazole (5p): Prepared from 3p and 4p. White solid, Yield: $72 \%$. ${ }^{1} \mathrm{H}$ NMR (400MHz, DMSO): $\delta 12.56$ (s, 1H), 8.36 (d, 1H, ArH), 7.59 (m, 1H, ArH), $7.52(\mathrm{~m}, 1 \mathrm{H}, \operatorname{ArH})$, 7.40 (m, 1H, ArH), 7.14 (m, 1H, ArH), 6.98 (m, 1H, ArH), 6.06 (s, 2H), 3.19 - 2.94 (m, 2H), 2.98 - 2.73 (m, 2H). ${ }^{13} \mathrm{C}$ NMR (100MHz, DMSO): $\delta$ 156.2, 151.1, 148.9, 147.1, 140.3, 137.0, 129.2, 127.3, 124.1, 121.1, 116.6, 115.8, 114.4, 111.9, 101.2, 70.4, 45, 39.9. Anal. Calcd for 
$\mathrm{C}_{18} \mathrm{H}_{14} \mathrm{ClN}_{3} \mathrm{O}_{3} \mathrm{~S}: \mathrm{C}-55.74 ; \mathrm{H}-3.64 ; \mathrm{N}-10.83 \%$; Found: $\mathrm{C}-55.44 ; \mathrm{H}-3.46 ; \mathrm{N}-10.23 \%$.

LCMS [M+1]: Calcd for $\mathrm{C}_{18} \mathrm{H}_{14} \mathrm{ClN}_{3} \mathrm{O}_{3} \mathrm{~S}: 388.84$, Found: 388.91.

5-[\{(6-chloro-1H-benzo[d]imidazol-2-yl)thio\}methyl]-3-(4-methoxyphenyl)-4,5-

dihydroisoxazole $(5 q)$ : Prepared from 3q and 4q. White solid, Yield: 76\%. ${ }^{1} \mathrm{H}$ NMR $(400 \mathrm{MHz}$, DMSO): $\delta 12.56(\mathrm{~s}, 1 \mathrm{H}), 7.57$ (d, 1H, ArH), 7.10 (m, 3H, ArH), 6.97 (d, 2H, ArH), 5.02 (m, $1 \mathrm{H}), 3.75(\mathrm{~s}, 1 \mathrm{H}, \mathrm{ArH}), 3.54(\mathrm{~m}, 3 \mathrm{H}), 3.50(\mathrm{~m}, 1 \mathrm{H}) .{ }^{13} \mathrm{C}$ NMR (100MHz, DMSO): $\delta 162.5$, 156.2, 147.1, 140.3, 137.0, 129.2, 128.7, 124.1, 122.1, 116.6, 115.8, 114.4, 70.4, 55.6, 45, 39.9. Anal. Calcd for $\mathrm{C}_{18} \mathrm{H}_{16} \mathrm{ClN}_{3} \mathrm{O}_{2} \mathrm{~S}$ : C - 57.83; $\mathrm{H}-4.31 ; \mathrm{N}-11.24 \%$; Found: $\mathrm{C}-57.21 ; \mathrm{H}$ - 4.27; $\mathrm{N}-11.07 \% . L C M S$ [M+1]: Calcd for $\mathrm{C}_{18} \mathrm{H}_{16} \mathrm{ClN}_{3} \mathrm{O}_{2} \mathrm{~S}: 374.07$, Found: 374.11 .

5-[\{(6-chloro-1H-benzo[d]imidazol-2-yl)thio\}methyl]-3-(4-nitrophenyl)-4,5dihydroisoxazole (5r):Prepared from 3r and 4r. Yellow solid, Yield: $71 \%$. ${ }^{1} \mathrm{H}$ NMR $(400 \mathrm{MHz}$, DMSO): $\delta 12.56$ (s, 1H), 8.36 (m, 1H ArH), 8.34 (d, 2H, ArH), 8.04 (d, 2H, ArH), 7.52 (m, $1 \mathrm{H}, \mathrm{ArH}), 7.12(\mathrm{~m}, 1 \mathrm{H}, \mathrm{ArH}), 3.91(\mathrm{~m}, 2 \mathrm{H}), 3.19-2.98(\mathrm{~m}, 2 \mathrm{H}), 2.94-2.78(\mathrm{~m}, 2 \mathrm{H}) .{ }^{13} \mathrm{C} \mathrm{NMR}$ (100MHz, DMSO): $\delta$ 156.2, 150.2, 147.1,140.3, 137.0, 136.5, 129.2, 127.7, 124.1, 116.7, 115.7, 70.4, 45, 39.9. Anal. Calcd for $\mathrm{C}_{17} \mathrm{H}_{13} \mathrm{ClN}_{4} \mathrm{O}_{3} \mathrm{~S}$ : C - 52.51; $\mathrm{H}-3.37 ; \mathrm{N}-14.41 \%$; Found: C - 52.43; H - 3.16; N - 8.14\%. LCMS [M+1]: Calcd for $\mathrm{C}_{17} \mathrm{H}_{13} \mathrm{ClN}_{4} \mathrm{O}_{3} \mathrm{~S}: 389.83$, Found: 389.92 .

\subsection{Biological Assay.}

\subsubsection{Assessment of Antioxidant Activity Using DPPH Assay.}

In vitro, antioxidant activities of all the synthesized compounds were evaluated by 1,1diphenyl-2-picryl-hydrazyl (DPPH) assay, which is a rapid and convenient technique for screening the antioxidant activities of the antioxidants. Standard was taken as $100 \%$ inhibition, and our compounds were compared with it. A higher percent value indicated greater antioxidant activity. Percent of inhibition values higher than $80 \%$ usually implied effective activities in antioxidant properties. The results were shown in Graph 1. Most of the synthesized compounds exhibited potent antioxidant activities. Compounds $\mathbf{5 a}, \mathbf{5 b}, \mathbf{5 c}, \mathbf{5 d}, \mathbf{5 g}$, 5k, 5n, and 5o showed excellent radical scavenging activities with percent inhibition above $70 \%$. Among all compounds $\mathbf{5 c}, \mathbf{5 g}$ and 50 showed excellent antioxidant activities with striking antioxidant values of higher than $80 \%$ inhibition, demonstrating greater antioxidant activities of these compounds in the assay. Based on the above observation, compounds having no substitution on benzimidazole moiety were found to be the most potent antioxidants than the compounds with electron-withdrawing $\mathrm{Cl}$ and $\mathrm{OCF}_{2} \mathrm{H}$ substituents whereas substitution of electron releasing groups at phenyl ring of isoxazoline showed least antioxidant activity and the activity enhanced with substitution of electron-withdrawing groups. From the above observation, we can easily notice that the electron releasing group on benzimidazole and electron-withdrawing group on the phenyl group of isoxazoline were found to be good antioxidants.

\subsubsection{Assessment of Antimicrobial Activity.}

All the synthesized compounds were evaluated for their antimicrobial activity using both Gram-negative (E.coli, P.aeruginosa, S.typhimurium), Gram-positive (B.subtilis, E.faecalis) bacteria, and Fungi (A.niger, C.albicans). A considerable number of compounds have been recognized exhibiting excellent to moderate inhibitory activity compared to standard percentage antimicrobial activitywas calculated $(\%)($ Table 1). Compounds without electron- 
withdrawing substituents on the benzimidazole ring exhibited better antimicrobial activity than the compounds with substitutions. Compounds with electron-withdrawing groups on the phenyl ring of isoxazoline $\mathbf{5 a}, \mathbf{5 c}, \mathbf{5 f}, \mathbf{5 g}, \mathbf{5 l}, \mathbf{5 m}$, and 50 showed excellent activity with lower MIC against bacterial strains. Other compounds 5a, 5c, 5f, 5g, 5h, 5, 5m, and 50 possessed good antifungal properties with lower MIC values. From the above discussion,5a and $\mathbf{5 f}$ were found to exhibit excellent antimicrobial activity.

\subsubsection{Haemolysis Assay.}

All the synthesized compounds were evaluated for their Haemolysis activity using Fresh chicken blood. Compounds 5a, 5c, 5d, 5h, 5m, 5p, and 5q have shown lower hemolysis on blood (Graph 2).

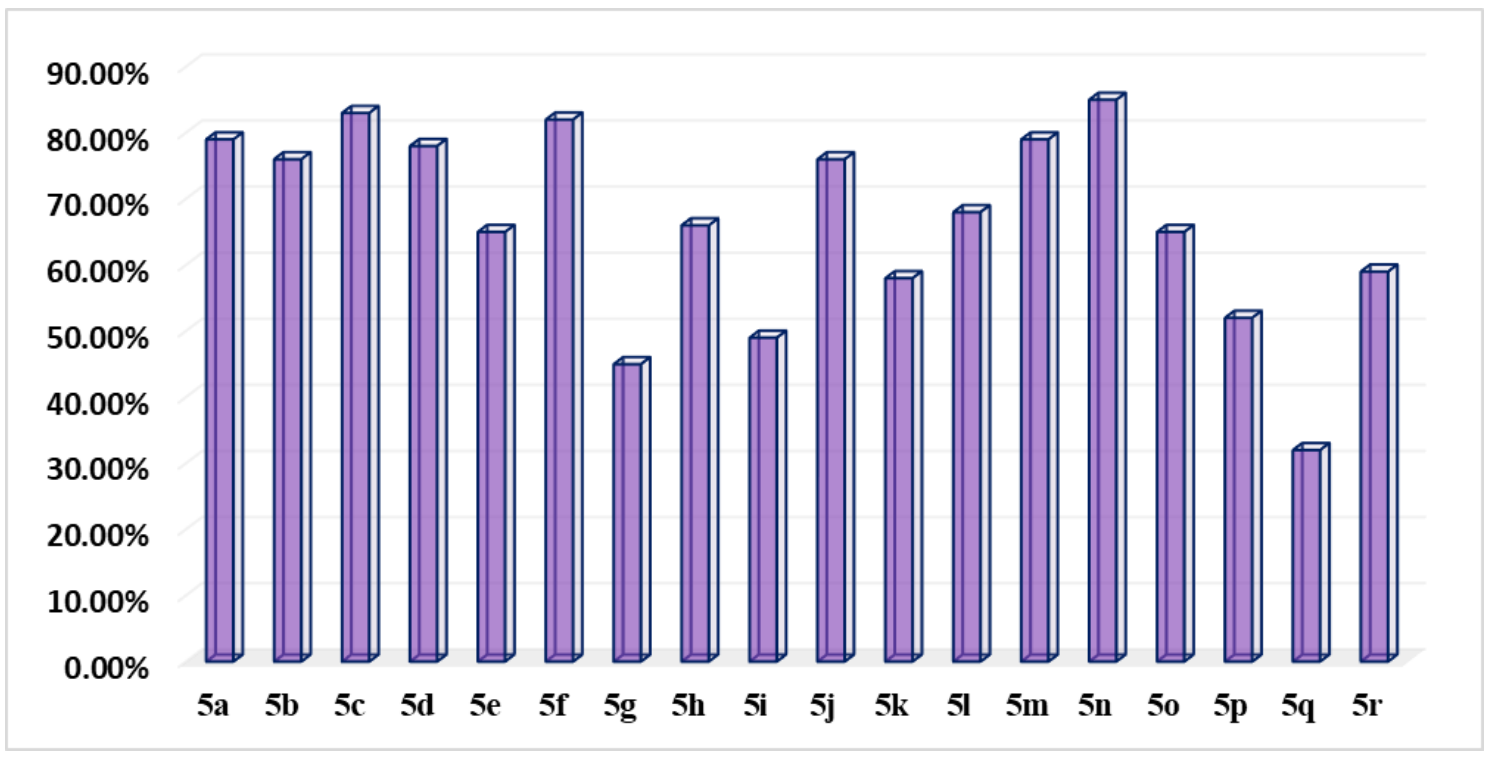

Graph 1. Antioxidant activity comparison of synthesized compounds (5a-5r).

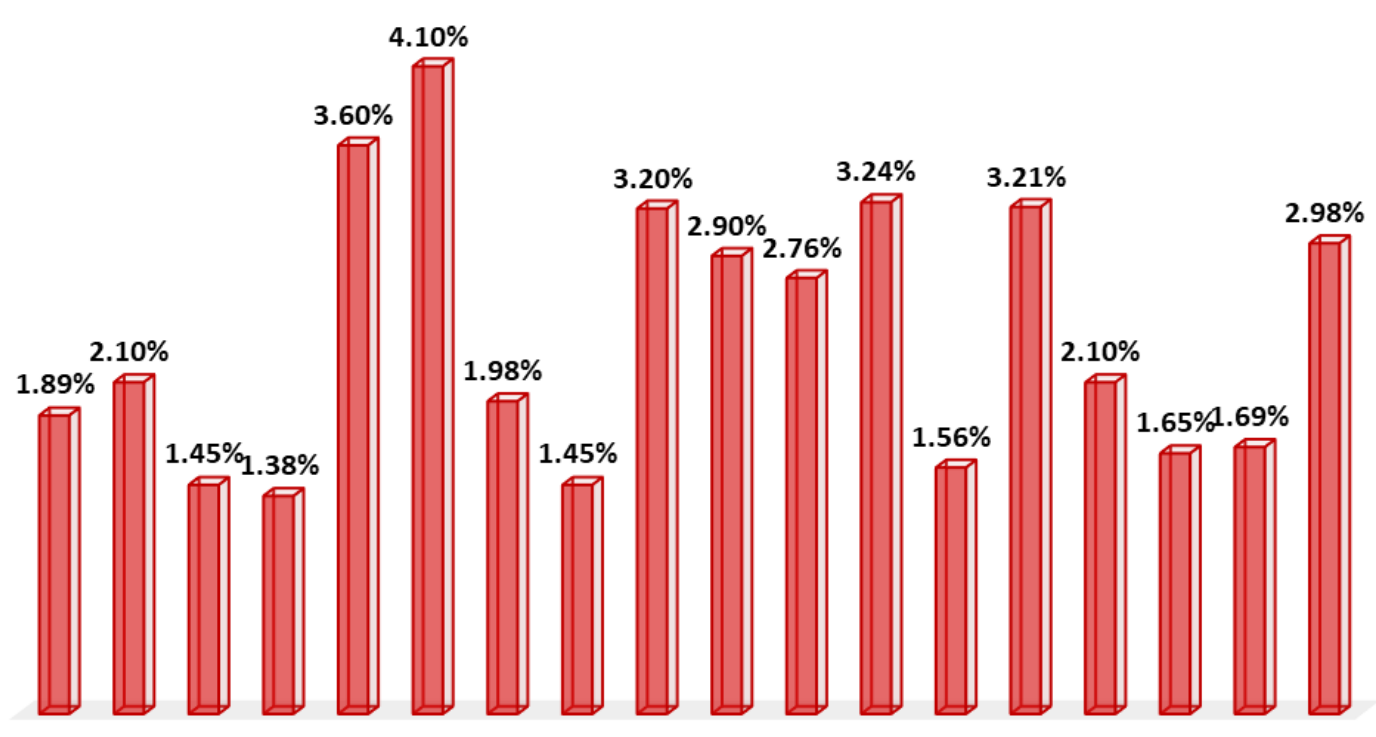

$\begin{array}{llllllllllllllllll}5 a & 5 b & 5 c & 5 d & 5 e & 5 f & 5 g & 5 h & 5 i & 5 j & 5 k & 5 l & 5 m & 5 n & 50 & 5 p & 5 q & 5 r\end{array}$

Graph 2. Hemolysis assay comparison of synthesized compounds (5a-5r). 
Table 1. Percentage antimicrobial activity (\%).

\begin{tabular}{|c|c|c|c|c|c|c|c|}
\hline \multirow{3}{*}{ Entry } & \multicolumn{5}{|c|}{ Bacteria } & \multirow{2}{*}{\multicolumn{2}{|c|}{ Fungi }} \\
\hline & \multicolumn{3}{|c|}{ Gram-negative } & \multicolumn{2}{|c|}{ Gram-positive } & & \\
\hline & E.coli & P.aeruginosa & S.typhimurium & B.subtilis & E.faecalis & A.niger & C.albicans \\
\hline $5 \mathrm{a}$ & 64 & 69 & 76 & 65 & 75 & 78 & 67 \\
\hline $5 b$ & 76 & 53 & 46 & 46 & 64 & 73 & 76 \\
\hline $5 \mathrm{c}$ & 54 & 47 & 34 & 47 & 56 & 76 & 34 \\
\hline $5 d$ & 82 & 76 & 72 & 77 & 74 & 56 & 66 \\
\hline $5 f$ & 49 & 52 & 76 & 65 & 77 & 35 & 48 \\
\hline $5 g$ & 78 & 43 & 65 & 83 & 82 & 67 & 82 \\
\hline $5 \mathrm{~h}$ & 43 & 68 & 73 & 75 & 56 & 74 & 56 \\
\hline $5 \mathrm{i}$ & 66 & 75 & 46 & 85 & 78 & 65 & 76 \\
\hline $5 j$ & 72 & 58 & 54 & 67 & 54 & 56 & 56 \\
\hline $5 n$ & 73 & 65 & 65 & 33 & 67 & 67 & 71 \\
\hline 50 & 52 & 57 & 67 & 48 & 45 & 54 & 46 \\
\hline $5 p$ & 55 & 85 & 46 & 71 & 65 & 45 & 73 \\
\hline $5 q$ & 65 & 67 & 46 & 76 & 36 & 65 & 65 \\
\hline $5 r$ & 80 & 56 & 64 & 43 & 81 & 56 & 55 \\
\hline Std & 100 & 100 & 100 & 100 & 100 & 100 & 100 \\
\hline
\end{tabular}

Std: Penicillin and Streptomycin

\section{Conclusions}

It is evident from the results that the compounds bearing electron-withdrawing groups $\mathrm{Br}$ and $\mathrm{NO}_{2}$ in phenyl ring of isoxazoline moiety and without substitution on benzimidazole moiety are better antioxidant agents. In conclusion, we have designed and synthesized three series of benzimidazole derived 2-isoxazolines derivatives as potential antioxidants, antibacterial agents with different groups in the phenyl ring. Of all the compounds synthesized, compounds $\mathbf{5 a}, \mathbf{5 c}$, and $\mathbf{5 f}$ exhibited stronger radical scavenging activity and antimicrobial activity with lower hemolysis.

\section{Funding}

This research received no external funding.

\section{Acknowledgments}

Authors NUK and SYK would like to acknowledge the University of Mysore for providing a laboratory facility, and the authors would also like to acknowledge IOE and UPE, University of Mysore, for instrumentation facilities.

\section{Conflicts of Interest}

The authors declare no conflict of interest.

\section{References}

1. Kotian, S.Y.; Mohan, C.D.; Merlo, A.A.; Rangappa, S.; Nayak, S.C.; Rai, K.L.; Rangappa, K.S. Small molecule based five-membered heterocycles: A view of liquid crystalline properties beyond the biological applications. J. Mol. Liq. 2020, 297, 1-30, https://doi.org/10.1016/j.molliq.2019.111686.

2. Kotian, S.Y.; Kudav, N.U.N.; Rai, K.M.L.; Byrappa, K. Synthesis of new series of 4, 5-dihydroisoxazole-5carboxylate derivatives for the study of their liquid crystalline properties. J. Chem. Sci. 2016, 128, 10331036, https://doi.org/10.1007/s12039-016-1114-0. 
3. Fritsch, L.; Baptista, L.A.; Bechtold, I.H.; Araújo, G.; Mandle, R.J.; Merlo, A.A. Isoxazoline-and isoxazoleliquid crystalline schiff bases: A puzzling game dictated by entropy and enthalpy effects. J. Mol. Liq. 2020, 298, https://doi.org/10.1016/j.molliq.2019.111750.

4. Kotian, S.Y.; Kudav, N.U.N.; Rai, K.M.L.; Byrappa, K. Synthesis of New Series of 4, 5-Dihydroisoxazole5-carbonitrile Derivatives for the Study of their Liquid Crystalline Properties. Materials Today: Proceedings 2016, 3, 4082-4087, https://doi.org/10.1016/j.matpr.2016.11.077.

5. Sathish, B.; Kavitha, R.; Sumana, Y.K.; Yashaswini, B.; Samudyata, C.P.; Kuriya, M.L.R.; Bharathi, P.S.Synthesis and Screening of Pro-apoptotic and Angio-inhibitory Activity of Novel Benzisoxazole Derivatives both In Vitro and In Vivo. Anticancer Agents in Medicinal Chemistry. 2019, 19, 827-839 https://doi.org/10.2174/1871520619666190114170621.

6. Tang, Z, Peng, Y, Liu, F. Design and synthesis of novel quinoline derivatives bearing oxadiazole, isoxazoline, triazolothiadiazole, triazolothiadiazine, and piperazine moieties. Journal of Heterocyclic Chemistry 2020, 1-9, https://doi.org/10.1002/jhet.3907.

7. Mohsen, G.L.; Abdula, A.M.; Jassim, A.M. Synthesis, Antimicrobial, Antioxidant and Docking Study of Novel Isoxazoline Derivatives. ACTA Pharmaceutica Sciencia 2018, 56, 7183,https://doi.org/10.23893/1307-2080.APS.05619.

8. Melek, G.; Serpil, E. Synthesis, Antioxidant Activity and Theoretical Investigation of Isoxazolines Derivatives of Monoterpenoids. Letters in Organic Chemistry 2019, 16, 501-510, https://doi.org/10.2174/1570178616666181226154540.

9. Weber, T.; Selzer, P.M. Isoxazolines: A Novel Chemotype Highly Effective on Ectoparasites. ChemMedChem 2016, 11, 270-276,https://doi.org/10.1002/cmdc.201500516.

10. Rodríguez Núñez, A.Y.; Gutíerrez, M.; Alzate-Morales, J.; Adasme-Carreño, F.; Güiza, M.F.; Bernal, C.C.; Bohórquez, R.R.A. Tetrahydroquinoline-Isoxazole/Isoxazoline Hybrid Compounds as Potential Cholinesterases Inhibitors: Synthesis, Enzyme Inhibition Assays, and Molecular Modeling Studies. International Journal of Molecular Sciences 2020, 21, 1-25,https://doi.org/10.3390/ijms21010005.

11. Guo, Y.; Zhang, Q.; Liu, Z.; Bao, C.; Fan, J.; Yang, R. Non-food bioactive products: Design and semisynthesis of novel (+)-nootkatone derivatives containing isoxazoline moiety as insecticide candidates. Industrial Crops and Products 2019, 140, 1-7,https://doi.org/10.1016/j.indcrop.2019.111706.

12. Kotian, S.Y.; Abishad, P.M.; Byrappa, K.; Rai, K.M.L. Potassium iodate (\$\$ $\$$ hbox $\left.\{\text { KIO }\}_{-}\{3\} \$ \$ K I O 3\right)$ as a novel reagent for the synthesis of isoxazolines: evaluation of antimicrobial activity of the products. Journal of Chemical Sciences 2019, 131,https://doi.org/10.1007/s12039-019-1622-9.

13. Morcoss, M.M.; Abdelhafez, E.S.M.N.; Abdel-Rahman, H.M.; Abdel-Aziz, M.; Abou El-Ella, D.A. Novel Benzimidazole/Hydrazone Derivatives as Promising Anticancer Lead Compounds: Design, Synthesis, and Molecular Docking Study. Journal of advanced Biomedical and Pharmaceutical Sciences 2020, 3, 45-52, https://dx.doi.org/10.21608/jabps.2020.21160.1064.

14. Scott, F.; Fala, A. M.; Pennicott, L. E.; Reuillon, T. D.; Massirer, K. B.; Elkins, J. M.; Ward, S. E. Development of 2-(4-pyridyl)-benzimidazoles as PKN2 chemical tools to probe cancer. Bioorg. Med. Chem. Lett. 202030127040 https://doi.org/10.1016/j.bmcl.2020.127040.

15. Francesconi, V.; Cichero, E.; Schenone, S.; Naesens, L.; Tonelli, M. Synthesis and Biological Evaluation of Novel (thio)semicarbazone-Based Benzimidazoles as Antiviral Agents against Human Respiratory Viruses. Molecules 2020, 25, https://doi.org/10.3390/molecules25071487.

16. Perin, N.; Škulj, S.; Martin-Kleiner, I.; Kralj, M.; Hranjec, M. Synthesis and Antiproliferative Activity of Novel 2-Substituted N-Methylated Benzimidazoles and Tetracyclic Benzimidazo [1,2-a]Quinolines. Polycyclic Aromatic Compounds 2020, 40, 343-354, https://doi.org/10.1080/10406638.2018.1441877.

17. Idris, Z.; Abbas, M.; Nadeem, H.; Khan, A.-u. The Benzimidazole Derivatives, B1 (N-[(1H-Benzimidazol2-yl)Methyl]-4-Methoxyaniline) and B8 (N-\{4-[(1H-Benzimidazol-2-yl)Methoxy $]$ Phenyl $\}$ Acetamide) Attenuate Morphine-Induced Paradoxical Pain in Mice. Frontiers in Neuroscience 2019, 13, https://doi.org/10.3389/fnins.2019.00101.

18. Wang, X.; Lou, K.; Song, X.; Ma, H.; Zhou, X.; Xu, H.; Wang, W. Mebendazole is a potent inhibitor to chemoresistant $\mathrm{T}$ cell acute lymphoblastic leukemia cells. Toxicology and Applied Pharmacology 2020, 396, https://doi.org/10.1016/j.taap.2020.115001.

19. Maghraby, M.T.E.; Abou-Ghadir, O.M.F.; Abdel-Moty, S.G.; Ali, A.Y.; Salem, O.I.A. Novel class of benzimidazole-thiazole hybrids: The privileged scaffolds of potent anti-inflammatory activity with dual inhibition of cyclooxygenase and 15-lipoxygenase enzymes. Bioorganic \& Medicinal Chemistry 2020, 28, https://doi.org/10.1016/j.bmc.2020.115403.

20. Zhang, X.; Zhang, C.; Tang, L.; Lu, K.; Zhao, H.; Wu, W.; Jiang, Y. Synthesis and biological evaluation of piperidyl benzimidazole carboxamide derivatives as potent PARP-1 inhibitors and antitumor agents. Chinese Chemical Letters 2020, 31, 136-140, https://doi.org/10.1016/j.cclet.2019.04.045.

21. Kumar, A.; Kumar, Y.; Sahu, J. K.; Kumar, S. Synthesis, Characterization and Antimicrobial Evaluation of Some N-Substituted Benzimidazole Derivatives. Curr. drug discov. technol. 2020, 17, 87-91, https://doi.org/10.2174/1570163815666180831164149. 
22. Majewsky, M.; Castel, D.; Le Dret, L.; Johann, P.; Jones, D.T.; Pfister, S.M.; Haefeli, W.E.; Burhenne, J. Systematic identification of suspected anthelmintic benzimidazole metabolites using LC-MS/MS. Journal of Pharmaceutical and Biomedical Analysis 2018, 151, 151-158, https://doi.org/10.1016/j.jpba.2017.12.056.

23. Yari, A.; Zolali, A.; Karimian, K. On the Mechanism of Formation and the Synthesis of Pantoprazole Sodium Sesquihydrate-Related Compound E: A Phantom Chemical Entity. ACS Omega 2019, 4, 737-744, https://doi.org/10.1021/acsomega.8b00845.

24. Welage, L.S.; Berardi, R.R. Evaluation of Omeprazole, Lansoprazole, Pantoprazole, and Rabeprazole in the Treatment of Acid-Related Diseases. Journal of the American Pharmaceutical Association (1996) 2000, 40, 52-62, https://doi.org/10.1016/S1086-5802(16)31036-1.

25. Kumar, P.; Singh, S.; Pratama, M.RF. Synthesis of some novel 1H-indole derivatives with antibacterial activity and antifungal activity. Letters in Applied NanoBioScience 2020, 9, 961967, https://doi.org/10.33263/LIANBS92.961967. 